\title{
LONCHAEIDAE ASSOCIADOS A FRUTOS DE NÊSPERA, ERYOBOTRIA JAPONICA (THUNB.) LINDLEY (ROSACEAE), COM A DESCRIÇÃO DE UMA ESPÉCIE NOVA DE NEOSILBA (DIPTERA: TEPHRITOIDEA)
}

\section{P.C.Strikis; A.P.Prado}

Universidade Estadual de Campinas, Instituto de Biologia, Departamento de Parasitologia, Distr. de Barão Geraldo, Campinas, SP, Brasil. E-mail: strikis@uol.com.br

\section{RESUMO}

A cultura da nêspera no Brasil está restrita praticamente ao Estado de São Paulo, e está novamente crescendo, contando com mais de 100 mil pés em produção, a maioria concentrado na região de Mogi das Cruzes. A diversidade de Lonchaeidae associada a esta fruta é apresentada neste artigo, juntamente com a descrição de uma nova espécie do gênero Neosilba, N. inesperata n. sp.

PALAVRAS-CHAVE: Nêspera, moscas-das-frutas, Lonqueídeos, espécie nova, Brasil.

\section{ABSTRACTS}

LONCHAEIDAE FOUND IN LOQUATS, ERYOBOTRIA JAPONICA (THUNB.) LINDLEY (ROSACEAE), WITH DESCRIPTION OF A NEW SPECIES OF NEOSILBA. Loquat production in Brazil is concentrated in the state of São Paulo, Brazil, and its economic importance has been growing during the last years, with more than 100 thousand trees under production, mostly in the region of Mogi das Cruzes, Brazil. This article presents the diversity of Lonchaeidae species associated with loquat and describes a new species of Neosilba, N. inesperata n. sp.

KEY WORDS: Loquat, fruit fly, lance flies, new species, Brazil.

\section{INTRODUÇÃO}

Algumas espécies do gênero Neosilba McAlpine são pragas importantes de plantações comerciais de frutas. UCHÔA-FERNANDESetal.(2003) observaram que, em plantações delaranja no cerrado de Mato Grosso do Sul, algumas espécies de Neosilba foram mais abundantes que espécies de tefritídeos. Araujo; ZucCHI (2002) observaram que, em certas condições, N. pendula (Bezzi) poderia tornar-se um invasor primário de plantações de acerola, Malphighiaemarginata (Wied.). LOURENÇão et al. (1996) mostraram a importância de se produzir novos clones de mandioca, Manihotesculenta Crantz, resistentes ao ataque de $N$. perezi (Romero \& Rupell) aos brotos apicais. No entanto, poucos trabalhos foram realizados até o momento com espécies deste gênero, pois a maioria ainda aguarda descrição.

McAlPINE; STEYSKAL (1982) observaram que o gêneroNeosilba compreendevários complexos deespécies, das quais algumas são espécies crípticas e que muitas ainda estão por serem descritas, pelo menos 60 , segundo estes autores. Neste mesmo trabalho, além de publicarem uma chave para a família Lonchaeidae, fizeram a redescrição de 12 espécies de Neosilba e descreveram outras três novas espécies. Atualmente, o gênero possui 16 espécies descritas (STRIKIS; PRADO, 2005). A identificação das espécies do gênero Neosilba é baseada na análise morfológica da genitália dos machos.

\section{Cultura da nêspera}

Deacordo com o Sindicato RuRAL de Jundiaí (2006), a maior produção de nêspera no Brasil está praticamente restrita ao Estado de São Paulo, com o maior pólo produtor localizado na região de Mogi das Cruzes. No passado chegou-se a mais de 200 mil pés, caindo posteriormente devido ao crescimento das cidades e à falta de mão de obra especializada. Entretanto, nos últimos anos, devido ao aumento da rentabilidade desta cultura houve uma retomada da produção; atualmente há mais de 100 mil pés em franca produção e um mercado em ascensão, o que está estimulando a produção também em outros estados do sudeste brasileiro, segundo o Sindicato Rural de Jundiaí.

A nêspera é originária do Japão, é uma fruta de clima temperado, que no Brasil, a partir da década de 1960 devido ao desenvolvimento, pelo Instituto Agro- 
nômico deCampinas, (IAC) devariedades adaptadas ao climalocal(Precoce deCampinas, Parmogi, Néctar deCristal, Mizauto, Mizumo e Centenária) teve o seu cultivo disseminado; estes cultivares apresentam alta produtividade e frutos de qualidade (OJIMA et al., 1999).

Opresente trabalho tem por objetivo fornecer uma chave para identificação dos espécimes do gênero Neosilba mais comumente encontrados em nêspera, além de descrever uma nova espécie deste gênero, facilitando desta forma, aos técnicos e pesquisadores que trabalham com este fruto, a identificação das espécies, permitindo uma avaliação mais rigorosa da importância econômica do gênero, bem como aprofundar os estudos da ecologia ebiologia do gênero Neosilba em frutos de nêspera.

\section{MATERIALEMÉTODOS}

Os espécimes obtidos de frutos de E.japonica foram colocados em solução de $\mathrm{NaOH} 10 \%$ a temperatura ambiente durante 24h. Após este período, as terminálias dos machos foram retiradas e dissecadas com o auxílio de um esteromicroscópio Zeiss.

As medições foram realizadas utilizando-se um fotomicroscópio AXIOPLAN e o programa computacional Image-Prolite versão 4.0 for Windows 95/NT/98.

\section{RESULTADOS}

Em frutos de nêspera (Eryobotria japonica Thunb. Lindley), juntamentecom esta nova espéciedeNeosilba, ocorreram também exemplares deN.pendula (Bezzi), N. certa (Walker), N. glaberrima (Wied.), N. zadolicha McAlpine \& Steyskal e N. bifida Strikis \& Prado.

\section{Neosilba zadolicha McAlpine \& Steyskal}

Neosilba zadolicha (McAlpine \& Steyskal, 1982): 127.2;(UCHÔA-FERNANDEsetal., 2002):515;(SANTOSetal., 2004): 653; (STRIKIS; PRADO, 2005): 1; (HAN; Ro, 2005): 420 .

Material examinado: $1160^{\pi} \sigma^{\pi}$, Cordeirópolis, Pedro Carlos Strikis, 8/1986;96 $0^{\pi} \sigma^{x}$, Louveira, Pedro Carlos Strikis, 8/1986; 14 o $\sigma^{\pi}$, São Bento do Sapucaí, Miguel Francisco de Souza Filho, 31/1/1996; $41 \sigma^{\pi} \sigma^{\pi}$, Monte Alegre do Sul, Pedro Carlos Strikis, 26/8/2003.

\section{Neosilbacerta(Walker)}

Anthomyia certa Walker, 1850-56: 364.

Neosilba certa: McAlpine \& Steyskal, 1982: 112; Souza et al., 2005: 639.
Lonchaea certa: Stein, 1901: 192.

Silba certa: Gaud \& Martorell, 1973: 249.

Lonchaea pendula: Bezzi, 1919: 249.

Material examinado: $117 \sigma^{\pi} \sigma^{\pi}$, Cordeirópolis, Pedro Carlos Strikis 8/1986; $21 \sigma^{\pi} \sigma^{\pi}$, Monte Alegre do Sul, Pedro Carlos Strikis, 26/8/2003; $62 \sigma^{x} \sigma^{x}$, Louveira, Pedro Carlos Strikis, 8/1986.

\section{Neosilbapendula (Bezzi)}

Lonchaea pendula Bezzi, 1919: 249.

Neosilba pendula: McAlpine \& Steyskal, 1982: 123; Araújo \& Zucchi, 2002: 91; Strikis \& Prado, 2005: 1; (Souza et al., 2005): 639.

Lonchaea glaberrima: R. von Ihering, 1905: 3; Hempel, 1605: 353; 1906: 208; Bezzi, 1910.

Lonchaea aenea: von Ihering, 1912; Tavares, 1915:52. Material examinado: $790^{\pi} \sigma^{x}$, Cordeirópolis, Pedro Carlos Strikis, 8/1986; $22 \sigma^{\pi} \sigma^{\pi}$, Louveira, Pedro Carlos Strikis 8/1986; $580^{x} \sigma^{x}$, Monte Alegre do Sul, Pedro Carlos Strikis, 18/7/2003.

\section{Neosilbaglaberrima(Wiedemann)}

Lonchaea glaberrima Wiedemann, 1830: 475.

Neosilba glaberrima: McAlpine \& Steyskal, 1982: 114; Souza et al., 2005: 639

Lonchaea pendula: Bezzi, 1919: 249.

Carpolonchaea pendula: Hennig, 1948: 360.

Silba pendula Korytkowski \& Ojeda, 1971: 112.

Material examinado: $94 \sigma^{\pi} \sigma^{\pi}$, Cordeirópolis, Pedro Carlos Strikis, 8/1986; 33 o $^{x} 0^{x}$ Louveira, Pedro Carlos Strikis 8/1986; $280^{\pi} \sigma^{x}$, Monte Alegre do Sul, Pedro Carlos Strikis, 14/8/2003.

\section{Neosilba bifida Strikis \& Prado, 2005: 1.}

Material examinado: $30^{\pi} 0^{\pi}$, São Bento do Sapucaí, Miguel Francisco de Souza Filho, 31/1/1996; $20^{\pi} \sigma^{\pi}$, Monte Alegre doSul, Pedro Carlos Strikis, 26/8/2003.

\section{Descrição Neosilba inesperata n.sp.}

Macho, tamanho do corpo 5,4 mm.

Cabeça: fronteafilada em direçãoà lúnula.Comprimento 1,95 mm; largura 1,39 mm razão comprimento/ largura 1,4. Lúnula com oito a nove setulas. Platô ocelar com quatro cerdas grandes; duas cerdas interocelares abaixodoplatôocelareduas outras sobreoplatôocelar. Antena com uma cerda proeminente no segundo segmento.Comprimentodeprimeiroflagelômero, $0,88 \mathrm{~mm}$; largura do primeiro flagelômero, 0,20 mm; razão comprimento/largura, 4,39. Arista comprida, 1,031 mm, e plumosa, mais comprida que o primeiro flagelômero; razão do comprimento da arista/comprimento do primeiro flagelômero, 1,16. Palpo amplo e largo. 
Tórax: mesonoto com 1,47 mm de comprimento; anepisterno com cinco cerdas grandes e um conjunto de três setas abaixo das cerdas, dorso do mesonoto setuloso, com 12 cerdas. Escutelo com margem lisa; duas cerdas marginais basais grandes e duas cerdas marginais apicais grandes, e um grupo de quatro setas marginais de cada lado; e duas setas entre as cerdas apicais.

Asas: hialinas, com $4,8 \mathrm{~mm}$ de comprimento e 2,06 $\mathrm{mm}$ de largura, veias amareladas e microtrichias pretas, razão comprimento/largura 2,33. Calípteras brancas, com franjas brancas e com um conjunto de seis a oito setas pretas, na dobra das calípteras.

Genitália do macho: epândrio comprido; mais comprido que largo; razão do comprimento/largura do epândrio, 2,67; com pelos longos (Fig. 1); que se alonga mais ao final da genitália,(Figs. 2e3). Surstilos com uma linha de prensisetas de cada lado, terminandonoúltimo quartoda genitália, próximoaocerco que é curto tanto em vista lateral quanto ventral (Figs. 1,2 e 3). Filamento do edeago longo e fino, terminando após os cercos, (Figs. 1 e 2). Base em forma de " $\mathrm{C}$ " do edeago com uma estrutura em forma de dentenolado convexo (Fig. 5); similar a Neosilba glaberrima, tal qual descrita por McAlPine; SteYsKal (1982). Parâmero com formato conspícuo (Fig. 4).

Fêmea: desconhecida.

Diagnose: a forma ea proporção do comprimento/ largura do epândrio, os pelos doepândrio,juntamente com a posição das prensiseta e forma do parâmero são as características mais importantes na identificação de N. inesperata n. sp.

Discussão taxonômica: $N$. inesperatan. sp.émuito similar a N. glaberrima McAlpine \& Steyskal, talvez seja a mais similar dentre as espécies de Neosilba. Externamente a única diferença aparente é a falta do arranjo característico dos pelos no final do abdome presente em N. glaberrima. A terminalia do macho possuia estrutura em forma de dentenolado convexo da base do edeago em forma de " $C$ ", que aparece também em $N$. glaberrima. No entanto, as diferençasna forma do parâmero, o arranjo das prensisetas no surstilos, bem como a diferença na proporção comprimento/largura do epândrio de $N$. insperata $n$. sp. em relação a N. glaberrima e a projeção do edeago, além do cerco, são suficientes para diferenciá-la de $N$. glaberrima.

Acredita-se que N. inesperata $n$. sp pertença ao mesmo complexo de espécies de $N$. glaberrima (Wied.).

Material examinado: holótipo; macho mantido em álcool 70\%, depositado no Museu de Zoologia da Universidade de São Paulo, obtido de nêspera, Eryobotria japonica (Thunb.) Lindl.; Monte Alegre do Sul (Estação Experimental do Instituto Agronômico de Campinas), latitude $22^{\circ} \mathrm{S} 40^{\prime} 50 "$, longitude: $46^{\circ} \mathrm{O} 40^{\prime} 45^{\prime \prime}$, altitude $760 \mathrm{~m}$, São Paulo, Brasil, Pedro Carlos Strikis, 15/7/2002.

Parátipos: dois machos mantidos em álcool $70 \%$, obtidos de laranja (Citrus sinensis (L.) Osbeck), Cordeirópolis(Estação Experimental doIAC), (latitude $22^{\circ} \mathrm{S} 28^{\prime} 50 "$, longitude $47 \mathrm{O}^{\circ} 27^{\prime} 27^{\prime \prime}$, altitude $660 \mathrm{~m}$ ), São Paulo, Brasil, Miguel Francisco de Souza Filho, 26/6/1996, depositados no mesmo museu.

Outros exemplares examinados de outros hospedeiros: $10^{\pi}$, Inga laurina Willd., Miguel Francisco de Souza Filho, Campinas, SP, (zona urbana), 30/ 1/2001, (esta amostra também continha exemplares de N. zadolicha e N. certa); $20^{\pi} 0^{\pi}$, Coffea arabica, Adalton Raga, Garça, SP, 20/3/1997, (esta amostra também continha exemplares de N. pendula); $1 \sigma^{x}$ Terminalia catappa L. (chapéu de sol), Adalton Raga, Assis, SP, zona urbana, 16/5/1996; 14 o $^{\pi} 0^{\pi}$, Malpighia punicifolia (acerola), Adalton Raga, Monte Mor, SP, 19/3/1999, [esta amostra continha também $10^{x}$ de N. glaberrima (Wied.)]; $20^{x} 0^{x}$, Averrhoa carambola L., Adalton Raga, Neves Paulista, SP, 22/ 10/1998; $3 \sigma^{\pi} \sigma^{\pi}, T$. catappa, Miguel Francisco de Souza Filho, Vinhedo, SP, 30/9/1999; $10^{\star}$, Psidium guajava L. (goiaba), Miguel Francisco de Souza Filho, Guairá, SP (zona urbana), 4/1/2001; $1 \sigma^{*}$, Passiflora allata Dryand (maracujá doce), Miguel Francisco de Souza Filho, Parelheiros, SP, em 23/ 3/1994; $1 \sigma^{x}$, Eugenia uniflora L. (pitanga), Miguel Francisco de Souza Filho, Bebedouro, SP, 7/12/ 1999; 1 ơ $^{\pi}$ Capsicum sp. (pimenta roxa), Miguel Francisco de Souza Filho, Ituverava, SP, 16/1/ 1997; $10^{\star}$, Citrus reticulata Blanco (laranja cravo), Miguel Francisco de Souza Filho, Regente Feijo, SP, 30/7/1996; $2 \sigma^{\pi} 0^{\pi}$, Myrcia sp. (maria - preta), Miguel Francisco de Souza Filho, Laboratório de Entomologia, Instituto Biológico, Campinas, SP, 17/4/2001; $1 \sigma^{\pi}$, Solanum paniculatum (jurubeba), Adalton Raga, Laboratório de Nematologia, Instituto Biológico, Campinas, SP, 14/1/1999; $1 \sigma^{x}$, Solanum gilo (jiló), Miguel Francisco de Souza Filho, de Estiva Gerbi, SP, 28/1/1998.

Distribuição geográfica: $N$. insperata n. sp. é conhecida do Estado de São Paulo dos Municípios de Regente Feijó, Assis, Neves Paulista, Garça, Bebedouro, Ituverava, Estiva Gerbi, Cordeirópolis, Campinas, Monte Mor, Vinhedo, Parelheiros e São Bento do Sapucaí. Ocupa uma ampla gama climática no Estado deSão Paulo, desde regiões quentes e secas (Bebedouro, Garça, Ituverava) até regiões frias e temperadas (São Bento do Sapucaí).

Hospedeiros: famílias de plantas hospedeiras de N. inesperata n. sp.: (Myrtaceae, Fabaceae/Mim., Rubiaceae, Combretaceae, Malpighiaceae, Oxalidaceae, Passifloraceae, Solanaceae, Rosaceae e Rutaceae). 

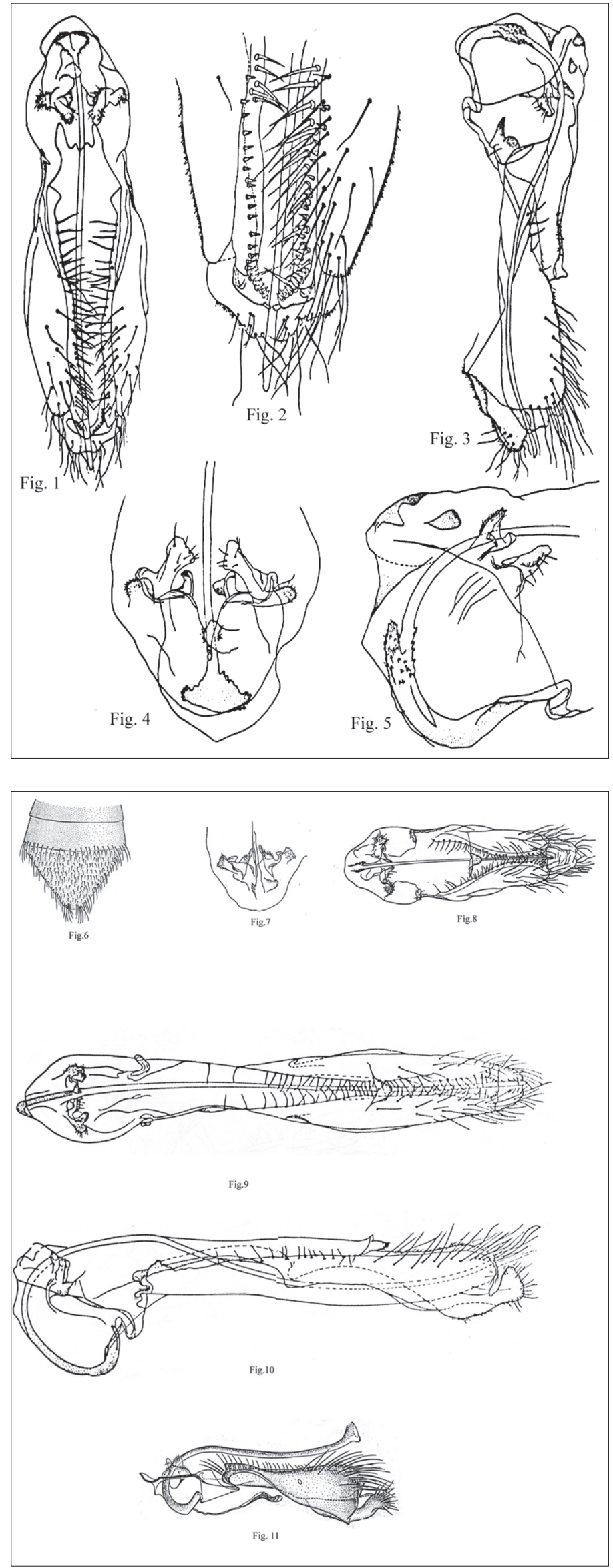

Prancha A - Fig. 1: Neosilba inesperata n. sp: Terminália macho de Neosilba inesperata sp.n. vista ventral (70X). Fig. 2: detalhe prensisetas e final do edeago (130X). Fig. 3: terminália macho vista lateral (70X). Fig. 4: detalhe parâmero vista ventral (130X). Fig. 5: detalhe base do edeago vista lateral (130X).

Prancha B - Fig.6: Vista dorsal do final do abdome de N. glaberrima mostrando arranjo característico dos pelos. Fig. 7: vista ventral com detalhe do parâmero do $\sigma^{T}$ de $N$. glaberrima. Fig. 8: vista ventral genitália do $\sigma^{x}$ de $N$. glaberrima. Fig. 9: vista ventral da genitália do $\sigma^{\pi}$ de N. zadolicha. Fig. 10: vista lateral da genitália do $o^{\top}$ de N. zadolicha. Fig. 11: vista lateral da genitália do $\sigma^{\pi}$ de $N$. pendula com edeago em destaque. Figuras copiadas de McAlPINE; STEYSKAL (1982) e de pranchas de Del Vechio (1991). 


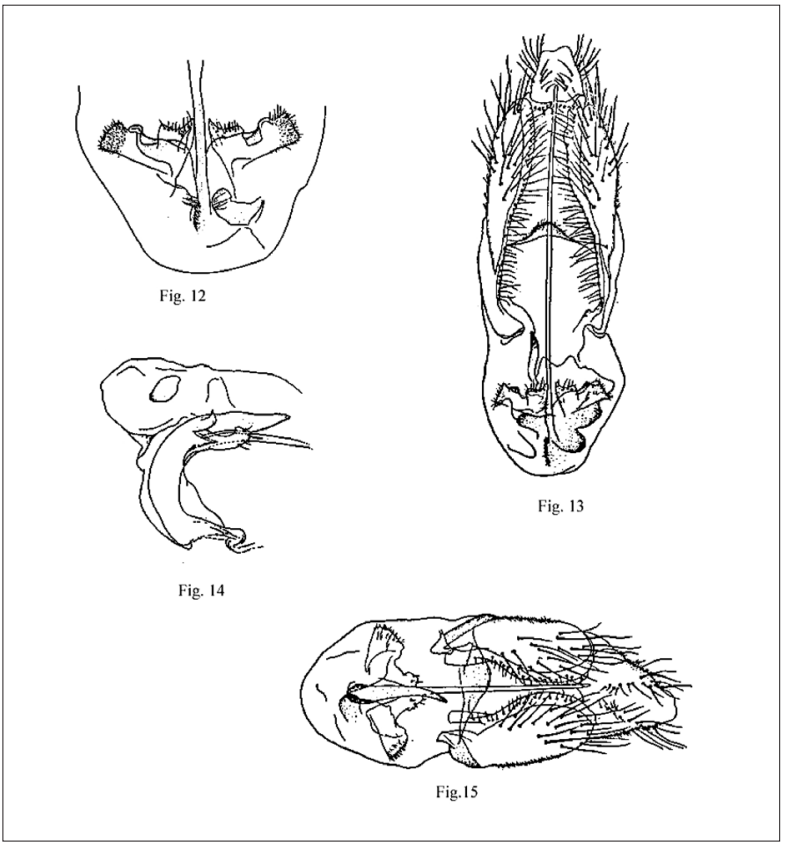

Prancha C - Fig. 12: vista ventral do parâmero em detalhe de N. certa. Fig. 13: vista ventral da genitália do $0^{x}$ de $N$. certa. Fig. 14: vista lateral com detalhe da estrutura em forma de espinha da base do edeago em forma de " $\mathrm{C}$ " de N. bifida. Fig. 15: Vista ventral da genitália do o de N. bífida. Figuras copiadas de McAlPine; STEYSKAL (1982) e de pranchas de DeL VeCHIO (1991).

Chave para identificação de espécies de Neosilba presentes em frutos Nêspera [adaptado de McAlpiNE; STEYSKal (1982) e STRIKIS; PRADO (2005)]:

1. Porção terminal do abdome com arranjo característico de pelos (Fig. 6) e forma do parâmero semelhante a uma lâmina (Fig.7), genitália do machoem vista ventral comona Figura 8-_—_- N. glaberrima (Wied.). Porção terminal do abdome com pelos distribuídos uniformemente, parâmero com alargamento em vista ventral2 . 2. Razão comprimento/largura maior da genitália do macho, superior a5(Fig.9), edeago finoe comprido(Fig.9), base em forma de "C" bastante pronunciada (Fig. 10)__ N. zadolicha (McAlpine \& Steyskal). Razão comprimento/largura maior do genitália do macho inferior a 5 3. 3. Edeago grosso e com alargamento no final, formando uma estrutura característica (Fig. 11)

Edeago fino sem diferenciaçãona porção final N.pendula (Bezzi). 4. Parâmero com forma próxima ao quadrado, com o ângulo do lado voltado ao edeago, formando uma estrutura em forma de espícula (Fig. 12), genitália do macho em vista ventral como na figura 13

Parâmero com forma arredondada, sem estruturas com formato de espículas N.certa(Walker). 5.Estrutura em forma deespinhoemergindoda porçãoterminal doladoconvexodabasedoedeagoem forma de "C", projetando-se até o início dos surstilos (Fig. 14), genitália do macho em vista ventral como Figura 15 N. bifida (Strikis \& Prado). Parâmero com forma arredondada conspícua (Fig. 4), edeago projetando-se além dos cercos (Fig. 2) N.inesperatan.sp.

\section{REFERÊNCIAS}

ARAÚJO, E.L.; ZUCCHI, R.A. Hospedeiros e níveis de infestação de Neosilba pendula (Bezzi) (Diptera: Lonchaeidade) na região de Mossoró-Açu, R.N. Arquivos do Instituto Biológico, São Paulo, v.69, n.2, p.91-94, 2002.

DEL VECCHIO, M.C. Família Lonchaeidae (Diptera: Acalyptratae): Ocorrência de espécies e respectivos hospedeiros em algumas localidades do Estado de São Paulo. 1991. 58f.
Dissertação (Mestrado em Biologia) - Universidade Estadual de Campinas, Campinas, 1991.

HAN, Y.H.; RO, K.E. Molecular phylogeny of the superfamily Tephritoidea (Insecta: Diptera): New evidence from the mitochondrial 12S, 16S, and COII genes. Molecular Phylogenetics Evolution, v.34, n.2, p.416-30, 2005.

LOURENÇÃO, A.L.; LORENZI, J.O.; AMBROSANO, G.M.B. Comportamento de clones de mandioca em 
relação a infestação por Neosilba perezi (Romero \& Rupell) (Diptera: Lonchaeidae). Scientia Agricola, v.53, p.304-308, 1996.

MCALPINE, J.F.; STEYSKAL, G.C. A revision of Neosilba McAlpine with a key to world genera of Lonchaeidae (Diptera). Canadian Entomologist, v.114, p.105-137, 1982.

OJIMA, M.; CAMPO DALL'ORTO, F.A.; BARBOSA, W.; MARTINS, F.P.; SANTOS, P.R. dos. Cultura de Nespereira. Campinas: Instituto Agronômico, 1999. 36p. (Boletim técnico, 185).

SANTOS, W.S.; CARVALHO, C.A.L.; MARQUES, O.M. Registro de Neosilba zadolicha McAlpine \& Steyskal (Diptera: Lonchaeidae) em umbú-cajá (Anacardiaceae). Neotropical Entomology, v.33, p. 653-654, 2004.

SINDICATO RURAL DE JUNDIAÍ. Nêspera Promissora fruta de produção intensiva. Disponível em: <http:/ / www.srjundiai.com.br>. Acesso em: 19 mar. 2006.

SOUZA, S.A.S.; RESENDE, A.L.S.; STRIKIS, P.C.; COSTA, J.R.; RICCI, M.S.F.; AGUIAR-MENEZES, E.
Infestação Natural de Moscas Frugívoras (Diptera: Tephritoidea) em Café Arábica, sob Cultivo Orgânico Arborizado e a Pleno Sol, em Valença, RJ. Neotropical Entomology, v.34, n.4, p.639-648, 2005.

STRIKIS, P.C.; PRADO, A.P. A new species of genus Neosliba (Diptera: Lonchaeidae). Zootaxa, v.828, p.1-5, 2005.

UCHÔA-FERNANDES, M.A.; OLIVEIRA, I.; MOLINA, R.M.S.; ZUCCHI, R.A. Species diversity of frugivorous flies (Diptera: Tephritoidea) from hosts in the cerrado of the State of Mato Grosso do Sul, Brazil. Neotropical Entomology, v.31, n.4, p.515-524, 2002.

UCHÔA-FERNANDES, M.A.; OLIVEIRA, I.; MOLINA, R.M.S.; ZUCCHI, R.A. Biodiversity of frugivorous flies (Diptera: Tephritoidea) captured in citrus groves, Mato Grosso do Sul, Brazil. Neotropical Entomology, v.32, n.2, p.239-246, 2003.

Recebido em 1/3/07 Aceito em 27/11/08 